\title{
Pre-eclampsia rates in the United States, 1980-2010: age-period-cohort analysis
}

\author{
@(థ) OPEN ACCESS
}

\author{
Cande V Ananth professor ${ }^{12}$, Katherine M Keyes assistant professor ${ }^{2}$, Ronald J Wapner professor ${ }^{1}$ \\ ${ }^{1}$ Department of Obstetrics and Gynecology, College of Physicians and Surgeons, Columbia University, 622 West 168th Street, New York, NY 10032 , \\ USA; ${ }^{2}$ Department of Epidemiology, Joseph L Mailman School of Public Health, Columbia University, New York, NY, USA
}

\begin{abstract}
Objective To estimate the contributions of biological aging, historical trends, and birth cohort effects on trends in pre-eclampsia in the United States.

Design Population based retrospective study.

Setting National hospital discharge survey datasets, 1980-2010, United States.

Participants 120 million women admitted to hospital for delivery.

Main outcome measures Temporal changes in rates of mild and severe pre-eclampsia in relation to maternal age, year of delivery, and birth cohorts. Poisson regression as well as multilevel age-period-cohort models with adjustment for obesity and smoking were incorporated.

Results The rate of pre-eclampsia was $3.4 \%$. The age-period-cohort analysis showed a strong age effect, with women at the extremes of maternal age having the greatest risk of pre-eclampsia. In comparison with women delivering in 1980, those delivering in 2003 were at 6.7-fold (95\% confidence interval 5.6-fold to 8.0-fold) increased risk of severe pre-eclampsia. Period effects declined after 2003. Trends for severe pre-eclampsia also showed a modest birth cohort effect, with women born in the 1970s at increased risk. Compared with women born in 1955, the risk ratio for women born in 1970 was 1.2 (95\% confidence interval 1.1 to 1.3 ). Similar patterns were also evident for mild pre-eclampsia, although attenuated. Changes in the population prevalence of obesity and smoking were associated with period and cohort trends in pre-eclampsia but did not explain the trends.

Conclusions Rates of severe pre-eclampsia have been increasing in the United States and age-period-cohort effects all contribute to these trends. Although smoking and obesity have driven these trends, changes in the diagnostic criteria may have also contributed to the age-period-cohort effects. Health consequences of rising obesity rates
\end{abstract}

in the United States underscore that efforts to reduce obesity may be beneficial to maternal and perinatal health.

\section{Introduction}

Pre-eclampsia is a pregnancy specific, multisystem syndrome characterized by reduced organ perfusion secondary to vasospasm and activation of the coagulation cascade. The condition complicates about $3-6 \%$ of pregnancies, with a 1.5 -fold to 2 -fold higher incidence in first pregnancies. ${ }^{1-4}$ The syndrome of pre-eclampsia is associated with high risks of preterm delivery, intrauterine growth restriction, placental abruption, and perinatal mortality. ${ }^{5-8}$ Additionally, pre-eclampsia serves as a sentinel marker for women who will experience premature cardiovascular and cerebrovascular diseases and other chronic illnesses later in life. ${ }^{90}$ Despite extensive research, the cause of pre-eclampsia remains elusive. ${ }^{11}$

The prevalence of pre-eclampsia has shown noticeable variation over time, leading to speculations that population level distribution of risk factors may have influenced these trends. Age-period-cohort analysis is a classic approach to understand how and why disease trends change over time. Pre-eclampsia has increased in both the youngest and the oldest women of reproductive age ${ }^{12}$ and the prevalence in the United States has increased from $2.5 \%$ in 1987 to $3.2 \%$ in $2004 .{ }^{13}$ This increase may be influenced by a variety of factors. For example, increases may be across age groups, indicative of a period effect, due to factors such as changes in the diagnostic criteria or earlier identification of symptoms during pregnancy. Although plausible, the influence of maternal birth cohorts remains unexplored. Women born in "older" cohorts may have different lifestyle factors (for example, smoking or illicit drug use) than women born in the more recent cohorts. Given that obesity is one of the strongest risk factors for pre-eclampsia ${ }^{14}{ }^{15}$ higher 
rates of pre-eclampsia in populations with higher proportions of obese women would be expected. If population level variation in obesity differs by both age and time period, as it does in the United States, ${ }^{16}$ then a birth cohort effect explaining trends over time may arise. Substantial research has indicated the presence of cohort effects in obesity rates over time, suggesting that such cohort effects may then extend to cohort effects in pre-eclampsia. ${ }^{17-19}$ In contrast, smoking during pregnancy is associated with a reduced risk of pre-eclampsia. ${ }^{20}$ The prevalence rate of smoking has been declining in the United States, ${ }^{21}$ and evidence indicates that both period and cohort effects may be underlying smoking trends in the United States. ${ }^{22}{ }^{23}$ Taken together, increases in obesity and decreases in smoking prevalence could lead to an increase in pre-eclampsia rates.

We examined pre-eclampsia rates for three different timescales, maternal age (time between maternal birth and disease diagnosis), period (time of occurrence of disease), and maternal birth cohort (time of mother's birth). The goal of this analysis was to understand the complex associations of maternal age, trends in prevalence, and cohort trajectories-all operating on a single timescale - on pre-eclampsia. We hypothesized that birth cohort effects may be responsible for increases in pre-eclampsia rates, given the increase in obesity among more recently born cohorts of women, as well as a decline in smoking rates. This population based study is based on births in the United States spanning three decades, 1980 through 2010.

\section{Methods}

\section{Study design and data sources}

We utilized the 1980-2010 national hospital discharge survey datasets, assembled by the US Centers for Disease Control and Prevention. Non-federal general and specialty short stay hospitals in the United States participated in the survey. ${ }^{24} \mathrm{We}$ restricted the analysis only to hospital admissions that resulted in childbirth. The data included in the analysis are representative of hospital discharges in the 50 states and District of Columbia that resulted in childbirth

Between 1980 and 1987 the survey used a two stage stratified sampling design; since 1988 the survey used a modified three stage design. ${ }^{25}$ The number of hospitals surveyed each year ranged between 400 and 558. Beginning in 2008, the number of hospitals chosen for the survey was reduced by half (roughly 200 to 300 hospitals.)

\section{Classification and diagnosis of pre-eclampsia}

The diagnosis of pre-eclampsia was based on the recommendations of the National High Blood Pressure Education Program Working Group. ${ }^{26}$ Mild pre-eclampsia was defined as a blood pressure $\geq 140 / 90 \mathrm{~mm} \mathrm{Hg}$ systolic/diastolic occurring at $\geq 20$ weeks' gestation and associated with the new onset of $\geq 300 \mathrm{mg}$ protein in a 24 hour maternal urine collection or $1+$ protein on a urine dipstick. Severe pre-eclampsia was defined as one of the following: blood pressures $\geq 160 \mathrm{~mm} \mathrm{Hg}$ systolic or $\geq 110 \mathrm{~mm} \mathrm{Hg}$ diastolic assessed at least twice over a six hour window; new onset proteinuria of $\geq 5000 \mathrm{mg}$ in a 24 hour period; oliguria $<500 \mathrm{~mL}$ in 24 hours; cerebral or visual disturbances, pulmonary edema, or cyanosis; epigastric/upper quadrant pain; impaired liver function, thrombocytopenia, or intrauterine growth restriction. ${ }^{27}$ Since the number of women with a diagnosis of eclampsia was too few, we combined eclampsia cases with those of severe pre-eclampsia. All diagnoses and procedures were coded based on the international classification of disease, ninth revision, clinical modification (ICD-9-CM). The ICD-9-CM codes that were used to abstract data on mild and severe pre-eclampsia and eclampsia were $642.4,642.5$, and 642.6 , respectively.

\section{Cohort composition}

We applied analytic weights contained in the national hospital discharge survey data based on the inverse of the probability that a US hospital was selected into the survey sample in each year of the data collection. Approximately 3.6 to 4.0 million hospital admissions resulted in childbirths each year, yielding a weighted total of about 120 million births between 1980 and 2010. These sampling weights were applied to produce national estimates and to consequently reflect generalizability of the findings. ${ }^{28}$ Of these births, we excluded women who were $\geq 46$ years of age $(n=54624)$, and women born either before 1940 $(n=33713)$ or during 1994 or later $(n=62$ 057). These exclusions were necessary to ensure stability in the estimation of age-period-cohort effects. We also excluded women with a diagnosis of chronic hypertension $(n=876484)$, chronic hypertension with superimposed pre-eclampsia $(n=288290)$, and gestational hypertension $(n=2398$ 404).

\section{Period and cohort level covariates}

We obtained the yearly prevalence of any current smoking as well as obesity (body mass index $\geq 30$ ) by maternal age among US women in the national health interview survey, ${ }^{29}$ a cross sectional, nationally representative household survey conducted between 1986 and 2010. Yearly estimates of obesity prevalence for women increased from $21.7 \%$ in 1986 to $36.0 \%$ in 2010 , while smoking among US adults decreased from $30.3 \%$ in 1987 to $18.7 \%$ in 2010 .

\section{Age-period-cohort models}

We modeled age-period-cohort effects (in single years) on pre-eclampsia from 1980 to 2010 . Since an individual's birth cohort is determined by the time period of study and the individual's age (that is, cohort=period-age), standard regression models to simultaneously estimate the additive effects of age, period, and cohort effects cannot achieve a unique solution. ${ }^{30}{ }^{31}$ We used two approaches to age-period-cohort analysis to provide a robust check on results. Our first approach to overcome the dependency was to estimate a variable for the overall linear trend in pre-eclampsia rates, which cannot be attributable uniquely to period or cohort effects but instead reflects the sum of linear period and cohort effects. This is referred to as a "drift" variable. ${ }^{32-34}$ Deviations from linearity (termed curvature), which can be uniquely attributable to period or cohort effects and are not dependent on any model constraint, are then estimated as period and cohort effects. We estimated pre-eclampsia rates using parametric smooth functions based on natural splines ${ }^{35}$ with five knots each for age, period, and cohort variables to detect non-linear effects. Modeling was carried out using the apc.fit function in the Epi package ${ }^{36}$ in the R program (version 2.14.2), with 1955 as the cohort referent and 1980 as the period referent group. We determined all models a priori and sequentially fit them beginning with age only, and then adding drift and other variables.

We also examined if other covariates affected the overall age-period-cohort analysis. Specifically, we used cross classified random effects, multilevel Poisson regression models. ${ }^{37}$ This model estimates a regression of log rates of pre-eclampsia on an intercept and age (categorised as $<20,20-24,25-29$, and $30-45$ years). We then estimated a second order regression of variation in the intercept across a set of period and cohort levels. We used two covariates: the prevalence of obesity and the 
prevalence of smoking by age among women. Period specific and cohort specific prevalence was entered in separate models due to collinearity and different years of data coverage (1986-2010 for obesity and 1987-2010 for smoking). We fit the multilevel models in SAS (version 9.3). The supplementary file provides details regarding the estimation of age-period-cohort models.

\section{Results}

\section{Trends in pre-eclampsia}

Figure $1 \Downarrow$ shows the prevalence of all pre-eclampsia and those of mild and severe pre-eclampsia between 1980 and 2010. For any pre-eclampsia, the rate increased from $3.4 \%$ in 1980 to $3.8 \%$ in 2010. This increase is driven by severe pre-eclampsia; the rate increased from $0.3 \%$ in 1980 to $1.4 \%$ in 2010 , a relative increase of $322 \%$. In contrast, the prevalence rate of mild pre-eclampsia declined by $19 \%$, from $3.1 \%$ in 1980 to $2.5 \%$ in 2010 (perhaps reflecting a shift from the mild to severe pre-eclampsia).

\section{Maternal age specific trends in pre-eclampsia}

Tables $1 \Downarrow$ and $2 \Downarrow$ show age specific trends in the prevalence of mild and severe pre-eclampsia, respectively; supplementary tables 1 and 2 show the corresponding data on the number of cases of mild and severe pre-eclampsia. The rate of mild pre-eclampsia decreased by period among women aged $<30$ years (for instance, among 15-19 year old women the rate dropped from $4.0 \%$ in 1980 to $2.8 \%$ in 2010), whereas the rate increased by period among older women (among 30-34 year old women, for instance, the rate increased from $1.7 \%$ in 1980 to $2.0 \%$ in 2010). In contrast, the rate of severe pre-eclampsia showed a consistent temporal increase by period within every maternal age group (table 2.)

\section{Age-period-cohort effects on pre-eclampsia}

Figures $2 \Downarrow$ and $3 \Downarrow$ show the model derived age-period-cohort effects on mild and severe pre-eclampsia, respectively. For mild pre-eclampsia, there is evidence of a cohort effect, with cohorts born around 1970 at an especially higher risk for mild pre-eclampsia (fig 2). For severe pre-eclampsia, women born in more recent cohorts have a higher risk (fig 3). Age effects show a J-shaped curve, with the highest risk at the extremes of the age distribution. There is evidence of an increasing period effect for both mild and severe pre-eclampsia, especially during the 1990s, and then a decrease in the period effect curvature in the late 2000s. This indicates that the slope of the period effect sharply increased in the 1980s and 1990s and began to decline thereafter.

For both mild and severe pre-eclampsia, the period effect estimate shows a decrease in curvature during a period when the underlying rate was increasing, especially for severe pre-eclampsia. This suggests that while rates of severe pre-eclampsia increased across women of all ages in the 1980s and 1990s, the rates increased primarily in younger women in the 2000s. This is consistent with the data shown in table 2 . For example, the rate of severe pre-eclampsia increased 5.7-fold between the 1957 and 1987 central birth cohorts for women aged 20-24 years but only 2.3-fold among those aged 40-45. Thus, younger birth cohorts of women primarily drove increases in the rate of severe pre-eclampsia in the 2000s.

\section{Multilevel modeling of period and cohort effects}

Supplementary figures 1 and 2 show the results of multilevel age-period-cohort models for mild and severe pre-eclampsia, respectively, with birth cohort and period as random intercept terms. This includes period specific and cohort specific prevalence of smoking and obesity in the United States as covariates of period and cohort effects. Results were similar to the analysis described in figure 2 . The overall period and cohort effects remained robust after adjustments for smoking and obesity prevalence by period and cohort, suggesting that these factors do not completely explain the observed period and cohort effects for both mild and severe pre-eclampsia.

\section{Discussion}

Temporal changes in rates of pre-eclampsia in the United States over three decades highlight four important findings. Although the rate of pre-eclampsia increased, two forces contributed simultaneously to this increase. Firstly, these data support our hypothesis that cohort effects in obesity may, at least partially, be responsible for increases in pre-eclampsia. Women born in the mid-1970s were at an increased risk for mild pre-eclampsia, whereas women born in the more recent periods showed an increased risk of severe pre-eclampsia, suggesting a birth cohort effect. Secondly, and contrary to our hypothesis, we observed a strong period effect, indicating that the rate of pre-eclampsia increased across all maternal ages. After the late 1990s and early 2000s, the magnitude of the period effect declined, which likely indicates that young women were at higher risk for developing pre-eclampsia. Thirdly, and as expected, these data showed increased pre-eclampsia rates among young and older women. Fourthly, declines in smoking prevalence in the United States are associated with increases in the period effect for mild and severe pre-eclampsia. In contrast, increases in the prevalence of obesity in the United States are associated with increases in the cohort effect, especially for severe pre-eclampsia. Overall, these results suggest that changes in the population proportion smoking during pregnancy impact on pre-eclampsia rates across age, whereas changes in obesity by cohort influence severe pre-eclampsia in younger cohorts only.

\section{Limitations of this study}

Geographic variations in the prevalence of pre-eclampsia in the United States are widespread, with states in the south showing higher rates than in other parts of the country ${ }^{13}$ and our analysis does not take into account the regional disparity. Confounding by maternal sociodemographic and behavioural factors may have influenced our findings to some extent. Some of the factors are either unavailable (for example, parity, body mass index, or smoking) or are poorly recorded (for example, a substantial proportion of births are missing data on race/ethnicity) in the survey. However, a subgroup analysis restricted to births where maternal race was recorded yielded results similar to the overall analysis (not shown). Nevertheless, static risk factors such as race/ethnicity and the average number of births per woman across the lifecourse are unlikely to influence the age-period-cohort trends since such factors have remained fairly constant over time. The possibility of women being pregnant more than once over the study period cannot be overlooked and our analyses do not take this into account.

\section{Strengths of this study}

This age-period-cohort analysis is the largest of any cohort studies (120 million births) to understand temporal changes in 
the prevalence of pre-eclampsia in the United States. The national hospital discharge survey data is arguably the most ideal of all data sources in the United States to study temporal changes of an obstetrical condition such as pre-eclampsia. The validity in the diagnosis of pre-eclampsia is considered excellent in the survey. ${ }^{38} 39$ The sensitivity and specificity for pre-eclampsia on the hospital discharge summaries (validated against chart abstraction of prenatal and medical and obstetrical records) were $88 \%$ and $95 \%$, respectively, while the positive and negative predictive values were $91 \%$ and $98 \%$, respectively. ${ }^{39}$ None the less, we acknowledge the possibility of some misclassification of pre-eclampsia cases in the national hospital discharge survey, although such misclassification is likely to be small and non-differential in nature.

Two population level trends are associated with these period and cohort changes: the prevalence of obesity (increasing throughout the study period) was associated with an increasing cohort effect for severe pre-eclampsia, and smoking (decreasing throughout the study period) was associated with an increasing period effect for both mild and severe pre-eclampsia, and an increasing cohort effect for mild pre-eclampsia. Overweight and obese women are susceptible to develop insulin resistance, ${ }^{40} 41$ which in turn is implicated as a strong (and modifiable) risk factor for pre-eclampsia. ${ }^{42}$ It is estimated that being overweight or obese accounts for $15-17 \%$ of cases of pre-eclampsia. ${ }^{43}$ Thus, the increases in the prevalence of obesity in the United States have likely resulted, at least partly, in a greater population burden of pre-eclampsia, especially among women born in more recent birth cohorts. Further, while smoking is a risk factor for many adverse pregnancy outcomes, ${ }^{44}$ it is associated with a lower risk for pre-eclampsia. ${ }^{20}{ }^{42}$ Decreases in the prevalence of smoking in the population may also contribute to a higher burden of pre-eclampsia. Another factor that may have contributed to the increase in pre-eclampsia includes metabolic disorders such as gestational diabetes, ${ }^{45}$ which has increased temporally in the United States. A subanalysis restricted to women without any diabetes (pregestational or gestational) was similar to the overall findings, providing reassurance to the stability of our results.

Our results show that the risk of pre-eclampsia for birth cohorts in the mid-1970s (women who would have borne children in the 1990s) is higher than expected. Given the long term health consequences of pre-eclampsia, ${ }^{9}{ }^{10}{ }^{46}$ women born in the mid-1970s cohorts, who are now approaching middle age, may be at particularly increased risk for adverse health outcomes through the life course. Furthermore, the offspring of these women should be carefully monitored for increases in adverse health outcomes given the associations between maternal obesity in pregnancy and adverse health in offspring.

\section{Maternal age, period, and birth cohort effects}

In addition to smoking and obesity, at least one other factor may partly help to explain the period and cohort effects we observed for trends in pre-eclampsia. Specifically, the decline in the period effect starting around 2003 may have to do with changes in diagnostic criteria for pre-eclampsia. In 2002, the criteria for the diagnosis of pre-eclampsia was revised by eliminating the use of incremental increases in blood pressure since this occurred in almost $25 \%$ of women ${ }^{47}$ Therefore, the new and stricter guidelines ${ }^{27}$ may have resulted in a decline in the observed period effect in pre-eclampsia since the early 2000s. In addition, an increased use of ultrasonography (to detect intrauterine growth restriction, a clinical criterion for diagnosing severe pre-eclampsia) may have also contributed to the temporal increase in severe pre-eclampsia. However, the effect of increased ultrasound use on temporal increase in severe pre-eclampsia is likely small.

There may be other unmeasured causes of changes in rates of pre-eclampsia, such as increased physician awareness or the prevalence of routine testing. Although data are unavailable to assess the contribution of such factors to trends in pre-eclampsia, we note that, similar to changes in diagnostic criteria, they would be unlikely to affect estimates of the cohort effect in pre-eclampsia.

A Norwegian study ${ }^{48}$ reported an increase in pre-eclampsia from $2.0 \%$ in $1967-74$ to $3.3 \%$ in 1998 . However, the rate increased to $4.4 \%$ in 1999 after the introduction of a revised notification form to record the diagnosis of pre-eclampsia, but the rate fell to $3.6 \%$ in 2008. Similar trends in pre-eclampsia rates have also been observed in Australia, Canada, Denmark, Scotland, and Sweden, and it has been speculated that an increase in rates of early elective obstetric interventions may have led to a decline in rates of pre-eclampsia since the early 2000 s. $^{49}$

\section{Conclusions}

Rates of severe pre-eclampsia are steadily increasing in the United States. The analyses show strong period effects for the temporal increase in pre-eclampsia up to the 1990s, but since the early 2000s, age and birth cohort effects appear to be primarily responsible for the increase. The trends for pre-eclampsia are concurrent to decreases in smoking prevalence and, more importantly, increases in the prevalence of obesity. These trends in the prevalence of pre-eclampsia seem biologically plausible, and may have social and behavioural underpinnings.

We thank Suneet Chauhan, Kirsten Lawrence Cleary, Alexander Friedman, Cynthia Gyamfi-Bannerman, and Anthony Vintzileos for their critical review and insightful comments that helped improve the manuscript.

Contributors: CVA had full access to all of the data in the study and takes responsibility for the integrity of the data and the accuracy of the data analysis. He also serves as the guarantor for the study. All authors conceived and designed the study, analysed and interpreted the data, and critically revised the manuscript for important intellectual content. CVA and KMK drafted the manuscript. CVA provided administrative, technical, and material support.

Competing interests: None of the authors report any potential conflict of interest.

Competing interests: All authors have completed the ICMJE uniform disclosure form at www.icmje.org/coi_disclosure.pdf and declare: no support from any organisation for the submitted work; no financial relationships with any organisations that might have an interest in the submitted work in the previous three years; no other relationships or activities that could appear to have influenced the submitted work.

\section{Ethical approval: Not required.}

Data sharing: No additional data available.

Transparency: CVA affirms that the manuscript is an honest, accurate, and transparent account of the study being reported; that no important aspects of the study have been omitted; and that any discrepancies from the study as planned have been explained.

1 Campbell DM, MacGillivray I, Carr-Hill R. Pre-eclampsia in second pregnancy. Br J Obstet Gynaecol 1985;92:131-40.

2 Basso $\mathrm{O}$, Christensen $\mathrm{K}$, Olsen J. Higher risk of pre-eclampsia after change of partner. An effect of longer interpregnancy intervals? Epidemiology 2001;12:624-9.

3 Skjaerven R, Wilcox AJ, Lie RT. The interval between pregnancies and the risk of preeclampsia. N Engl J Med 2002;346:33-8.

4 Hernandez-Diaz S, Toh S, Cnattingius S. Risk of pre-eclampsia in first and subsequent pregnancies: prospective cohort study. BMJ 2009;338:b2255.

5 Conde-Agudelo A, Belizan JM, Diaz-Rossello JL. Epidemiology of fetal death in Latin America. Acta Obstet Gynecol Scand 2000;79:371-8. 


\section{What is already known on this topic}

Pre-eclampsia is associated with high risks of preterm delivery, intrauterine growth restriction, placental abruption, and perinatal mortality Although rates of pre-eclampsia have increased, the extent to which maternal age, period, and birth cohorts may have contributed to this increase remains unknown

\section{What this study adds}

The rate of severe pre-eclampsia has been increasing in the United States, and period and cohort effects have both contributed to these trends

These trends also seem to be influenced by secular changes in increasing obesity and declining smoking prevalence rates Despite the contribution of maternal age and birth cohorts, changes in the diagnostic criteria of pre-eclampsia may have also contributed to these trends

6 Zhang J, Troendle JF, Levine RJ. Risks of hypertensive disorders in the second pregnancy. Paediatr Perinat Epidemiol 2001;15:226-31.

Goldenberg RL, Culhane JF, lams JD, Romero R. Epidemiology and causes of preterm birth. Lancet 2008;371:75-84

8 Ananth $\mathrm{CV}$, Basso O. Impact of pregnancy-induced hypertension on stillbirth and neonatal mortality. Epidemiology 2010;21:118-23

9 Irgens HU, Reisaeter L, Irgens LM, Lie RT. Long term mortality of mothers and fathers after pre-eclampsia: population based cohort study. BMJ 2001;323:1213-7.

10 Ray JG, Vermeulen MJ, Schull MJ, Redelmeier DA. Cardiovascular health after maternal placental syndromes (CHAMPS): population-based retrospective cohort study. Lancet 2005;366:1797-803

11 Ness RB, Sibai BM. Shared and disparate components of the pathophysiologies of fetal growth restriction and preeclampsia. Am J Obstet Gynecol 2006;195:40-9.

12 Zhang J, Zeisler J, Hatch MC, Berkowitz G. Epidemiology of pregnancy-induced hypertension. Epidemiol Rev 1997;19:218-32.

13 Wallis AB, Saftlas AF, Hsia J, Atrash HK. Secular trends in the rates of preeclampsia eclampsia, and gestational hypertension, United States, 1987-2004. Am J Hypertens 2008;21:521-6.

14 Bodnar LM, Ness RB, Markovic N, Roberts JM. The risk of preeclampsia rises with increasing prepregnancy body mass index. Ann Epidemiol 2005;15:475-82.

15 Getahun D, Ananth CV, Oyelese Y, Chavez MR, Kirby RS, Smulian JC. Primary preeclampsia in the second pregnancy: effects of changes in prepregnancy body mass index between pregnancies. Obstet Gynecol 2007;110:1319-25.

16 Flegal KM, Carroll MD, Ogden CL, Curtin LR. Prevalence and trends in obesity among US adults, 1999-2008. JAMA 2010;303:235-41.

17 Reither EN, Hauser RM, Yang Y. Do birth cohorts matter? Age-period-cohort analyses of the obesity epidemic in the United States. Soc Sci Med 2009;69:1439-48.

18 Robinson WR, Keyes KM, Utz RL, Martin CL, Yang Y. Birth cohort effects among US-born adults born in the 1980s: foreshadowing future trends in US obesity prevalence. Int $J$ Obes (Lond) 2013;37:448-54

19 Robinson WR, Utz RL, Keyes KM, Martin CL, Yang Y. Birth cohort effects on abdominal obesity in the United States: the silent generation, baby boomers and generation X. Int J Obes (Lond) 2013;37:1129-34

20 England L, Zhang J. Smoking and risk of preeclampsia: a systematic review. Front Biosc 2007;12:2471-83.

21 Garrett BE, Dube SR, Trosclair A, Caraballo RS, Reshacek TF; Centers for Disease Control and Prevention (CDC). Cigarette smoking-United States, 1965-2008. MMWR Surveill Summ 2011;60(Suppl):109-13.

22 Keyes KM, March D, Link BG, Chilcoat HD, Susser E. Do socio-economic gradients in smoking emerge differently across time by gender? Implications for the tobacco epidemic from a pregnancy cohort in California, USA. Soc Sci Med 2013;76:101-6.

23 Preston $\mathrm{SH}$, Wang $\mathrm{H}$. Sex mortality differences in the United States: the role of cohort smoking patterns. Demography 2006:43:631-46.

24 DeFrances CJ, Cullen KA, Kozak LJ. National Hospital Discharge Survey: 2005 annual summary with detailed diagnosis and procedure data. Vital and health statistics Series 13, Data from the National Health Survey 2007(165):1-209.

25 Kozak LJ, Owings MF, Hall MJ. National Hospital Discharge Survey: 2001 annual summary with detailed diagnosis and procedure data. Vital and health statistics Series 13, Data from the National Health Survey 2004(156):1-198.

26 Report of the National High Blood Pressure Education Program Working Group on High Blood Pressure in Pregnancy. Am J Obstet Gynecol 2000;183:S1-22.

27 ACOG practice bulletin. Diagnosis and management of preeclampsia and eclampsia. № 33, Jan 2002. Obstet Gynecol 2002;99:159-67.

28 Dennison C, Pokras R. Design and operation of the National Hospital Discharge Survey: 1988 redesign. Vital and health statistics Series 1 , Programs and collection procedures 2000(39):1-42.

29 National Health Interview Survey. National Center for Health Statistics, Centers for Disease Control and Prevention. www.cdc.gov/nchs/nhis.htm. 2013.
30 Glenn ND. Cohort analysis. 2nd ed Sage, 2005

31 Yang Y. Age/period/cohort distinctions. In: Markides KS, ed. Encyclopedia of health and aging. Sage, 2007

32 Carstensen B. Age-period-cohort models for the Lexis diagram. Stat Med 2007;26:3018-45

33 Clayton D. Schifflers E. Models for temporal variation in cancer rates. II: age-period-cohort models. Stat Med 1987;6:469-81.

34 Holford TR. Analysing the temporal effects of age, period and cohort. Stat Methods Med Res 1992;1:317-37.

35 Hastie TJ, Tibshirani RJ. Generalized Additive Models. Chapman and Hall, 1990

36 Carstensen B, Plummer M, Hils M, Laara E. Epi: a package for statistical analysis in epidemiology (R package version 1.1.34). 2012. http://CRAN.R-project.org/package=Epi.

37 Yang Y, Land K. Chapter 7. Mixed effects models: hierarchical APC-cross-classified random effects models (HAPC-CCREM), Part I: the basics. In: Yang Y, Land K, eds. Age-period-cohort analysis: new models, methods, and empirical applications. Chapman and Hall/CRC Interdisciplinary Statistics, 2013

38 Klemmensen AK, Olsen SF, Osterdal ML, Tabor A. Validity of preeclampsia-related diagnoses recorded in a national hospital registry and in a postpartum interview of the women. Am J Epidemiol 2007:166:117-24.

39 Yasmeen S, Romano PS, Schembri ME, Keyzer JM, Gilbert WM. Accuracy of obstetric diagnoses and procedures in hospital discharge data. Am J Obstet Gynecol 2006;194:992-1001

40 Mangos GJ, Spaan JJ, Pirabhahar S, Brown MA. Markers of cardiovascular disease risk after hypertension in pregnancy. $J$ Hypertens 2012;30:351-8.

41 Ness RB, Roberts JM. Heterogeneous causes constituting the single syndrome of preeclampsia: a hypothesis and its implications. Am J Obstet Gynecol 1996;175:1365-70.

42 Engel SM, Janevic TM, Stein CR, Savitz DA. Maternal smoking, preeclampsia, and infant health outcomes in New York City, 1995-2003. Am J Epidemiol 2009;169:33-40.

43 Catov JM, Ness RB, Kip KE, Olsen J. Risk of early or severe pre-eclampsia related to pre-existing conditions. Int J Epidemiol 2007;36:412-9.

44 Cnattingius S. The epidemiology of smoking during pregnancy: smoking prevalence, maternal characteristics, and pregnancy outcomes. Nicotine Tob Res 2004;6(Suppl 2): S125-40.

45 Bombard JM, Dietz PM, Galavotti C, England LJ, Tong VT, Hayes DK, et al. Chronic diseases and related risk factors among low-income mothers. Matern Child Health $\mathrm{J}$ 2012;16:60-71.

46 Sibai BM. Intergenerational factors: a missing link for preeclampsia, fetal growth restriction, and cardiovascular disease? Hypertension 2008:51:993-4.

47 Roberts JM, Pearson GD, Cutler JA, Lindheimer MD. Summary of the NHLBI working group on research on hypertension during pregnancy. Hypertens Pregnancy 2003;22:109-27.

48 Klungsoyr K, Morken NH, Irgens L, Vollset SE, Skjaerven R. Secular trends in the epidemiology of pre-eclampsia throughout 40 years in Norway: prevalence, risk factors and perinatal survival. Paediatr Perinat Epidemiol 2012;26:190-8.

49 Roberts CL, Ford JB, Algert CS, Antonsen S, Chalmers J, Cnattingius S, et al. Population-based trends in pregnancy hypertension and pre-eclampsia: an international comparative study. BMJ Open 2011;1:e000101.

\section{Accepted: 9 October 2013}

\section{Cite this as: BMJ 2013:347:f6564}

This is an Open Access article distributed in accordance with the Creative Commons Attribution Non Commercial (CC BY-NC 3.0) license, which permits others to distribute, remix, adapt, build upon this work non-commercially, and license their derivative works on different terms, provided the original work is properly cited and the use is non-commercial. See: http://creativecommons.org/licenses/by-nc/3.0/. 


\section{Tables}

Table 1| Rates of mild pre-eclampsia (\%) by selected maternal age, period, and central maternal birth cohorts in United States, 1980 to 2010

\begin{tabular}{|c|c|c|c|c|c|c|c|}
\hline \multirow[b]{2}{*}{ Period of birth } & \multicolumn{6}{|c|}{ Maternal age (years) } & \multirow[b]{2}{*}{ Central birth cohor } \\
\hline & $15-19$ & $20-24$ & $25-29$ & $30-34$ & $35-39$ & $40-45$ & \\
\hline & & & & & & 2.3 & 1937 \\
\hline & & & & & 1.6 & 3.7 & 1942 \\
\hline & & & & 1.7 & 1.8 & 5.8 & 1947 \\
\hline & & & 2.4 & 1.5 & 1.6 & 1.5 & 1952 \\
\hline & & 2.6 & 1.9 & 1.4 & 2.1 & 2.4 & 1957 \\
\hline & 4.0 & 2.5 & 1.3 & 1.7 & 1.3 & 4.6 & 1962 \\
\hline 1980 & 3.4 & 2.4 & 2.1 & 1.5 & 1.8 & 5.1 & 1967 \\
\hline 1985 & 1.7 & 2.4 & 1.7 & 2.0 & 1.9 & & 1972 \\
\hline 1990 & 3.6 & 2.5 & 1.9 & 2.0 & & & 1977 \\
\hline 1995 & 3.0 & 2.2 & 1.6 & & & & 1983 \\
\hline 2000 & 2.5 & 2.4 & & & & & 1987 \\
\hline 2005 & 2.8 & & & & & & \\
\hline 2010 & & & & & & & \\
\hline
\end{tabular}

The cohort follows from lower left to upper right, going diagonally.

See supplementary table 1 for number of mild pre-eclampsia cases for each of age-period cross classifications. 
Table 2| Rates of severe pre-eclampsia (\%) by selected maternal age, period, and central maternal birth cohorts in United States, 1980 to 2010

\begin{tabular}{|c|c|c|c|c|c|c|c|}
\hline \multirow[b]{2}{*}{ Period of birth } & \multicolumn{6}{|c|}{ Maternal age (years) } & \multirow[b]{2}{*}{ Central birth cohort } \\
\hline & $15-19$ & $20-24$ & $25-29$ & $30-34$ & $35-39$ & $40-45$ & \\
\hline & & & & & & $-^{*}$ & 1937 \\
\hline & & & & & 0.2 & - $^{*}$ & 1942 \\
\hline & & & & 0.2 & 0.2 & 0.9 & 1947 \\
\hline & & & 0.1 & 0.4 & 0.6 & 0.8 & 1952 \\
\hline & & 0.3 & 0.4 & 0.5 & 0.7 & 0.8 & 1957 \\
\hline & 0.6 & 0.6 & 0.5 & 0.9 & 0.5 & 0.9 & 1962 \\
\hline 1980 & 0.9 & 0.7 & 0.6 & 0.7 & 0.7 & 2.1 & 1967 \\
\hline 1985 & 0.7 & 0.3 & 1.0 & 0.9 & 1.3 & & 1972 \\
\hline 1990 & 1.1 & 0.9 & 0.9 & 1.0 & & & 1977 \\
\hline 1995 & 1.4 & 0.9 & 1.4 & & & & 1983 \\
\hline 2000 & 1.0 & 1.7 & & & & & 1987 \\
\hline 2005 & 1.9 & & & & & & \\
\hline 2010 & & & & & & & \\
\hline
\end{tabular}

The cohort follows from lower left to upper right, going diagonally.

Supplementary table 2 shows number of severe pre-eclampsia cases for each of age-period cross classifications.

*Number of severe pre-eclampsia cases $<25$ to produce stable estimates of rates. 


\section{Figures}

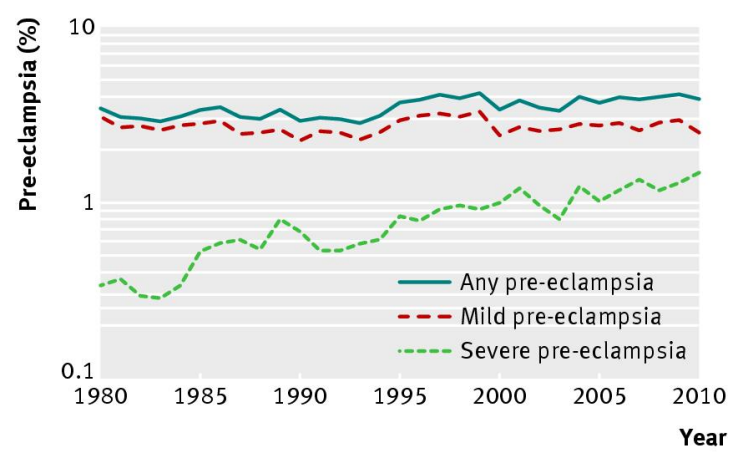

Fig 1 Temporal changes in prevalence of pre-eclampsia: United States, 1980 to 2010

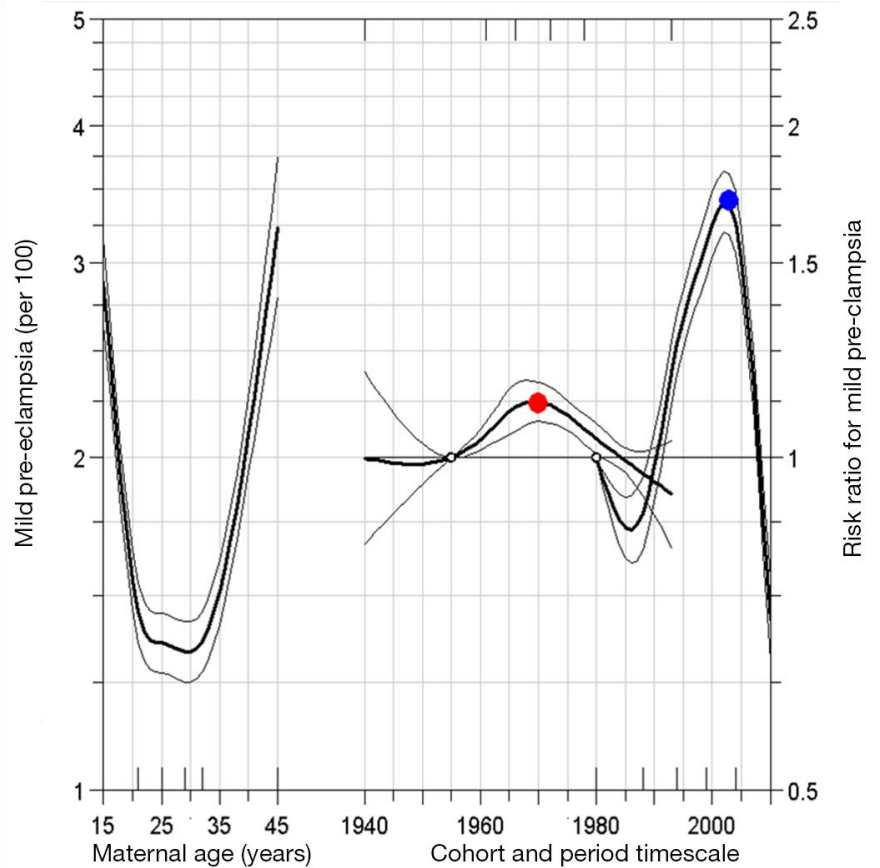

Fig 2 Age-period-cohort influences on trends in mild pre-eclampsia: United States, 1980 to 2010. Open circles refer to reference group for birth cohort and period. Adjusted risk ratio (red dot) for 1970 birth cohort (relative to reference cohort $1955)$ is 1.2 (95\% confidence interval 1.1 to 1.2$)$. Similarly, risk ratio (blue dot) for 2003 birth period (relative to reference period 1980) is 1.7 (95\% confidence interval 1.6 to 1.8). Small hash marks on bottom x-axis pertain to knot locations for birth cohort, and hash marks on top axis refer to knot locations for period. Estimates for period effect are second order derivatives, indicating that slope of period effect for mild pre-eclampsia has been decelerating since 2003 


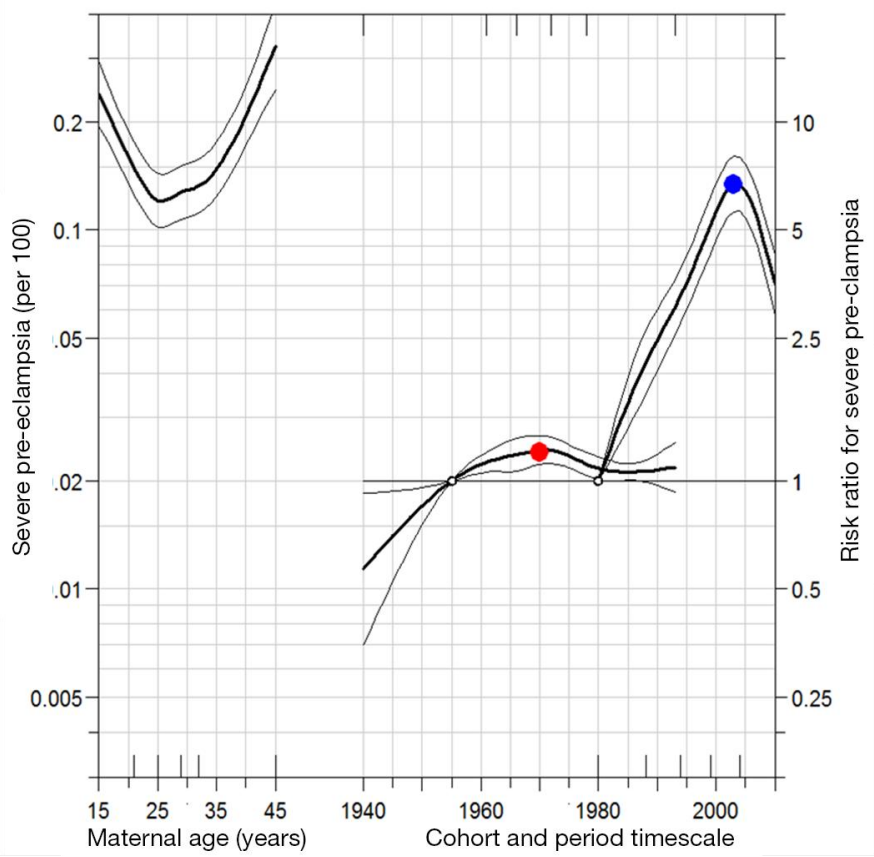

Fig 3 Age-period-cohort influences on trends in severe pre-eclampsia: United States, 1980 to 2010. Open circles refer to reference group for birth cohort and period. Adjusted risk ratio (red dot) for 1970 birth cohort (relative to reference cohort 1955 ) is 1.2 (95\% confidence interval 1.1 to 1.3). Similarly, risk ratio (blue dot) for 2003 birth period (relative to reference period 1980) is 6.7 (95\% confidence interval 5.6 to 8.0). Small hash marks on bottom $x$ axis pertain to knot locations for birth cohort, and hash marks on top axis refer to knot locations for period. Estimates for period effect are second order derivatives, indicating that slope of period effect for severe pre-eclampsia has been decelerating since 2003 